\title{
Glue Impressions of Maize Leaves and Their Use in Classifying Mutants
}

Lindy A. Allsman, Raquel N. Dieffenbacher and Carolyn G. Rasmussen*

Department of Botany and Plant Sciences, Center for Plant Cell Biology, Institute for Integrative Genome Biology, University of California, Riverside, USA

*For correspondence: carolyn.rasmussen@ucr.edu

[Abstract] Maize cells are surrounded by cell walls that can be easily visualized using a simple protocol outlined below. A glue impression is an imprint of maize epidermal cells on a glass slide made using a cyanoacrylate-based glue (super glue) that produces a permanent record of the leaf (Wilson et al., 1981). Although we demonstrate use of this protocol with Zea mays (maize) leaves, the protocol can be easily adapted to other sturdy leaves. Briefly, the leaf blade is mounted in liquid cyanoacrylate glue on a glass slide and held still until the glue dries. The leaf is removed and the resulting glue impression is observed using a standard light microscope. The glue impression can be used to analyze epidermal cells for defects in patterning or division. Types of cell defects that are easily detected include cytokinesis defects, symmetric and asymmetric division plane orientation defects (Gallagher and Smith, 1999; Sylvester and Smith, 2009), lobing or crenulation defects (Frank and Smith, 2002) and aberrantly expanded cells (Reynolds et al., 1998; Hunter et al., 2012). Importantly, glue impressions are a simple, fast method to make a permanent record of maize leaves that require very little sample processing or special equipment. Keywords: Maize, Cytokinesis, Cell wall, Lobing, Cell expansion, Glue impression

\section{Materials and Reagents}

1. Frosted glass microscope slides

2. Lab gloves

3. Ultra fine point permanent marker

4. Paper towels

5. Plastic protective cover (clear plastic autoclave bag)

6. Resealable plastic bag

7. Maize leaves

8. Liquid cyanoacrylate glue (Loctite Super Glue, catalog number: 234796)

9. Tap water

\section{Equipment}

1. Microscope slide box

2. A light microscope with at least $100 x$ total magnification

3. Digital microscope camera (AmScope MD130) 


\section{Software}

1. Software (AmScope) to take images with the digital microscope camera

\section{Procedure}

1. Grow maize under standard greenhouse or field conditions until desired developmental stage. We use leaf two after the fourth leaf emerged to examine juvenile leaves (Figure 1). Alternatively, older adult leaves can also be used to examine cell patterning, although in many mutants with stomatal defects, phenotypes are less severe in adult leaves. Regardless of leaf used, it is important to use the identical leaf number across samples. Leaf number is determined by counting the first emerging leaf (oldest, leaf 1 ) and counting up. Note that epidermal crenulations are not fully complete until after the leaf is fully expanded.

2. Collect $\geq 3 \mathrm{~cm} X \geq 1 \mathrm{~cm}$ portion of maize leaf blade avoiding the midrib by tearing or cutting.

3. Write a unique number on the leaf (leaf ID) on collected leaf blade with black permanent marker and place in a resealable sandwich-type plastic bag with a small amount $(\sim 5 \mathrm{ml})$ of water. Collected leaf samples can stay fresh up to one week at $4-10{ }^{\circ} \mathrm{C}$.

4. In the lab, lay down a non-fibrous, plastic cover, such as a clear plastic autoclave bag, to protect your lab bench.

5. Put on lab gloves and set out frosted slides, super glue, permanent marker, and paper towels.

6. Take out fresh wet leaves from the bag, and blot to dry on a paper towel. Drying the sample prevents water bubbles in the glue impression, see Notes section below for more.

7. Open the liquid super glue container. If it is glued shut, use scissors to carefully remove the glue.

8. Apply 4 to 5 thin strips of liquid super glue onto a glass slide (Figure $2 \mathrm{~A}$ ). This number of glue impressions easily fits on one slide.

9. Remove a leaf strip and press the leaf down with at least a few millimeters of leaf tissue hanging off the glass-slide edge for easier removal (Figure 2B). We typically use the abaxial side, see Data analysis section below for more.

10. Using a permanent marker write the unique identifier for the sample (leaf ID, for example) on the frosted section of the slide (Figure $2 \mathrm{C}$ ). If samples are close to each other, number the glass slide next to the individual leaves.

11. After each leaf has been mounted in super glue, use a weight (such as a full box of glass slides) to flatten and dry the glue impression.

12. Wait around $3 \mathrm{~min}$ for the glue to dry. If the leaf is removed before the glue dries, the impression can look stretched or wrinkled (Figure 3B). You can wait up to two days for the glue to dry, but after that the leaf becomes difficult to remove.

13. Slowly pull off the fresh leaf making sure super glue does not come off the slide. If the glue is peeling away from the slide, try removing the leaf from the other side. Keep samples if you need to re-do the glue impressions. 
14. View the slide under a light microscope. We use a final magnification of $100 x$ for observation, and take photos using a digital microscope camera and software to classify types of mutants.

15. Examples of leaf two abaxial glue impressions display normal patterning (Figure 2D), cytokinesis defects (Figure 2E), asymmetric division defects (Figure 2F), and overexpanded cells (Figure 2G).

16. Examples of leaf 8 glue impressions from the adaxial side show a macrohair (Figure $2 \mathrm{H}$, caret), bulliform cells (Figure 2l, asterisk) and microhair (Figure 2l, caret). Normal and abnormal stomatal cell complexes are shown in (Figure 2J, left and right, respectively. Arrow shows abnormal subsidiary cell division).

17. Glue impressions can be stored in a microscope slide box for several years for later reexamination.

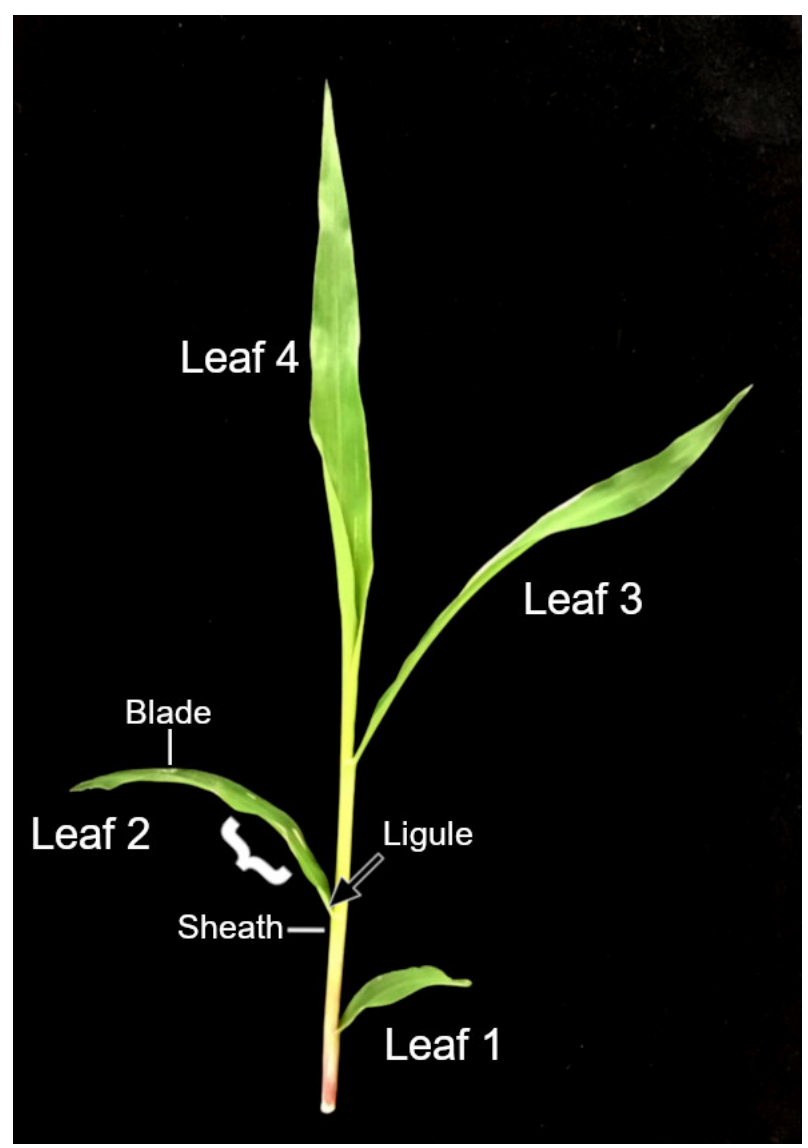

Figure 1. Maize leaf numbering. As leaves emerge from the maize plant they are numbered beginning from leaf 1 and continuing numerically. Sheath, blade, and ligule are indicated. Bracket indicates the area typically sampled for glue impressions. 


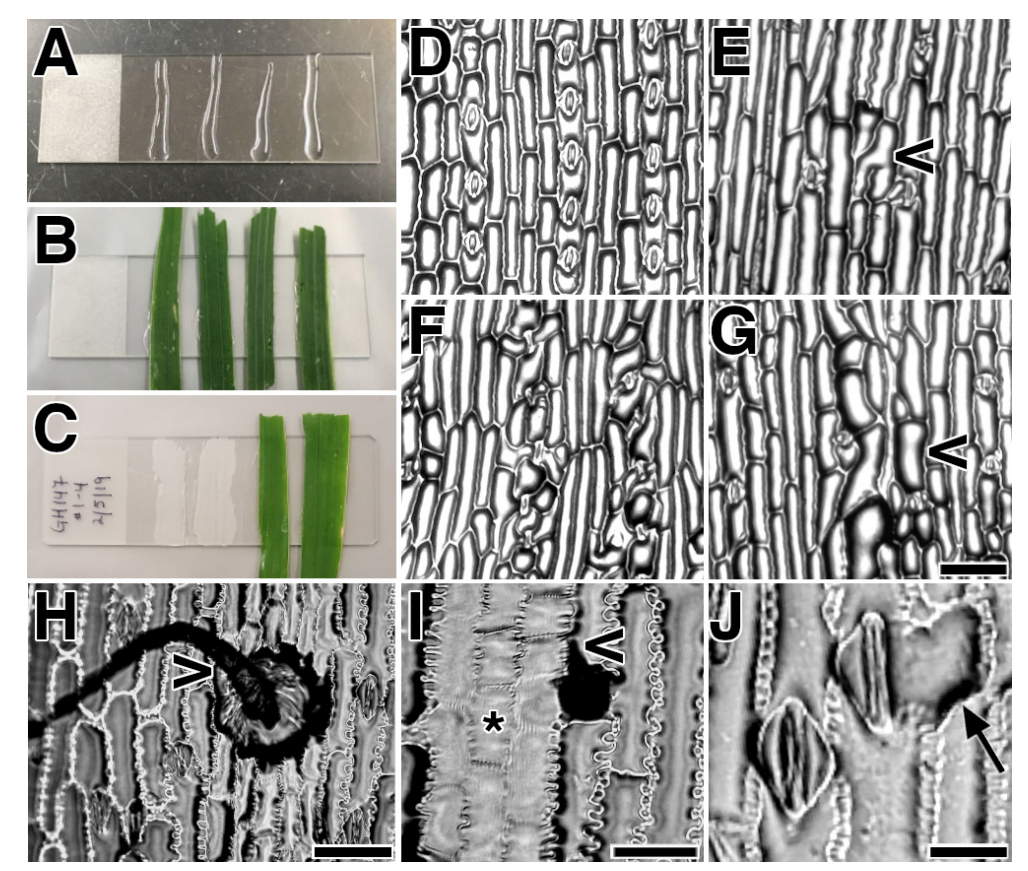

Figure 2. Maize glue impressions. A. Liquid super glue is applied in thin strips along the clear section parallel to the frosted section of a glass slide. B. Maize leaves are placed on glue strips. C. Labeled slide indicates leaf ID, leaf number, and date. Two leaves are removed from glue strips to display imprint used for image analysis. D. Wild-type maize (B73) mature abaxial leaf cell impressions are in a brick-like, organized pattern. E. Mutant maize cell walls displaying cell wall stub characteristic of a cytokinesis defect (indicated with caret). F. Mutant maize cell walls show asymmetric division plane orientation defects. G. Mutant maize cell walls displaying excessively enlarged cells characteristic of a "warty" defect (indicated with caret). H. Leaf 8 Adaxial wild-type maize leaf macrohair (indicated with caret). I. Leaf 8 Adaxial wild-type maize leaf bulliform cells, with characteristic wrinkled morphology (indicated with asterisk) and microhair (indicated with caret). J. Leaf 8 stomatal cells complex with normal appearance (left), and aberrant subsidiary cell (right, indicated with arrow). Scale bars, $100 \mu \mathrm{m}$ (D, E, F, G), $75 \mu \mathrm{m}$ (H), $50 \mu \mathrm{m}(\mathrm{I}), 15 \mu \mathrm{m}(\mathrm{J})$.

\section{Data analysis}

1. Several different cell types can be found on the maize epidermis depending on the developmental stage and leaf side. To reduce complexity, we usually screen abaxial juvenile leaves, as pavement cell lobing is reduced and the abaxial side lacks macrohairs and cork, bulliform, and silica cells. However, in leaf 2 , occasional ( $2 \%$ ) asymmetric positioning of subsidiary cell divisions can be seen in B73 and other wild-type cells. Screening adult leaves is also valuable for identifying defects, particularly in asymmetric division, lobing and expansion. Pavement cells have extensive lobing or crenulations. Differences in mature adaxial and abaxial leaf sides can also be readily observed: there are typically no macrohairs or bulliform cells on the abaxial surface. On the 
adaxial surface, there are more cell types, such as macrohairs (Figure $2 \mathrm{H}$, caret), bulliform cells (Figure 2l, asterisk), and microhairs (Figure 2l, caret).

2. When quantifying defects in subsidiary cell divisions, take multiple (from $\geq 3$ plants) random and unbiased images of your glue impressions of the mutant and a developmentally matched (same leaf and position from the ligule) sibling or near isogenic wild-type sample. Label each subsidiary cell in your sample set, count the total number, and count how many aberrant subsidiary cells from mutant and wild-type samples (> 75 subsidiary cells per individual). Divide the number of aberrant subsidiary cells by the total, and multiply by 100 to calculate percentage of abnormal stomatal subsidiary cells of your samples (Zhang et al., 2012).

3. The veins of maize leaves appear in glue impressions as raised columns of cells. Note that epidermal pavement cell patterning around vasculature is distinct from other pavement cells. We typically avoid analyzing cells for division plane or expansion defects near vasculature and leaf margins.

\section{Notes}

1. Use care to avoid unintentional contact with super glue. It is difficult to remove.

2. Wet leaf samples create non-ideal glue impressions, containing streaks and bubbles. Properly drying the sample before making the impression is recommended.

3. When taking pictures using the AM Scope MD130 digital camera, remove one eyepiece of the light microscope by twisting gently and placing in a foam case to prevent damage. Next, insert the camera into the eyepiece. With the camera USB cable attached to your computer you can then use the AM Scope software to take pictures. Under the "Camera List" window click on "MD130" to view a glue impression mounted on the light microscope. We look through the standard eyepiece to find the area of interest using the 10x objective and then switch to the $4 x$ objective for taking the photo. The camera adds $2 x$ magnification when viewing and taking photos. The area of interest shown in the software window is focused and properly lit by adjusting illumination and focus on the microscope. Other color features can be adjusted using the software such as brightness, contrast, and saturation. The quick save function can be used by clicking on the settings icon, navigating to the desired directory, naming the sample with the unique identifier and pressing "OK". To take a picture, press "snap", and click on the icon "quick save" which will save to your desired directory using the unique identifier and will automatically and sequentially label the files.

4. Air bubbles occur when the super glue container is old or has been agitated: prevent shaking or dropping super glue container or use a fresh container (Figure 3A).

5. Removing leaves before the glue dries, not pressing the sample under adequate weight, or sliding the sample around in the glue before drying can result in a wrinkled appearance of the imprint (Figure 3B). If a wrinkled glue impression is formed, re-do the glue impression with either the same sample or a fresh sample. 


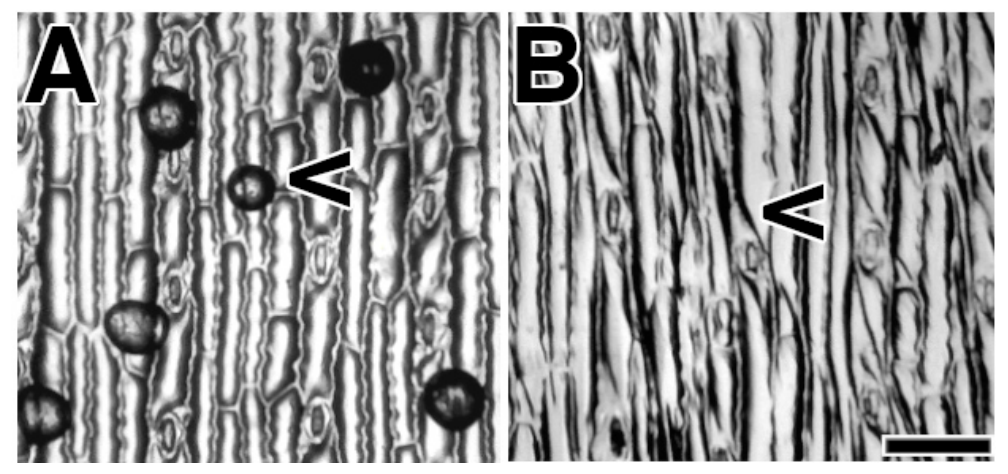

Figure 3. Common problems observed with maize leaf glue impressions. A. Air bubbles on maize leaf cell impression caused by shaking the glue (caret). B. Wrinkled appearance of maize glue impression caused by removing the leaf before the glue fully dried (caret). Light microscope imaging using AM Scope digital camera. Scale bar, $100 \mu \mathrm{m}$ (A, B).

\section{Acknowledgments}

Special thanks to Professor Michelle Facette (University of Massachusetts, Amherst), and Rasmussen Lab members for protocol edits. Special thanks to Professors Amanda J. Wright (University of North Texas), Anne W. Sylvester (University of Wyoming), and Laurie G. Smith (University of California, San Diego), for instructions and demonstrations of this technique. Thanks to Dr. David Carter (Microscopy Core Facility, University of California, Riverside) for use of his stage micrometer for calibration. NSF-MCB \#1716972 is gratefully acknowledged for funding.

Competing interests: All authors declare no competing interests.

\section{$\underline{\text { References }}$}

1. Frank, M. J. and Smith, L. G. (2002). A small, novel protein highly conserved in plants and animals promotes the polarized growth and division of maize leaf epidermal cells. Curr Biol 12(10): 849-853.

2. Gallagher, K. and Smith, L. G. (1999). Discordia mutations specifically misorient asymmetric cell divisions during development of the maize leaf epidermis. Development 126(20): 4623-4633.

3. Hunter, C. T., Kirienko, D. H., Sylvester, A. W., Peter, G. F., McCarty, D. R. and Koch, K. E. (2012). Cellulose Synthase-Like D1 is integral to normal cell division, expansion, and leaf development in maize. Plant Physiol 158(2): 708-724.

4. Reynolds, J. O., Eisses, J. F. and Sylvester, A. W. (1998). Balancing division and expansion during maize leaf morphogenesis: analysis of the mutant, warty-1. Development 125(2): 259268.

5. Sylvester, A. W. and Smith, L. G. (2009). Cell biology of maize leaf development. In: Handbook of Maize: Its Biology. In: Bennetzen, J. L. and Hake, S.C. (Eds.). Springer, New York. pp. 179203. 
6. Wilson, C. K. S. L., Pusey, P. L. and Otto, B. E. (1981). Plant epidermal sections and imprints using cyanoacrylate adhesives. Can J Plant Sci 61: 781-783.

7. Zhang, X., Facette, M., Humphries, J. A., Shen, Z., Park, Y., Sutimantanapi, D., Sylvester, A. W., Briggs, S. P. and Smith, L. G. (2012). Identification of PAN2 by quantitative proteomics as a leucine-rich repeat-receptor-like kinase acting upstream of PAN1 to polarize cell division in maize. Plant Cell 24(11): 4577-4589. 